\title{
LA DINÁMICA DE LA CONSTRUCCIÓN \\ EN BOGOTÁ 1995-2013*
}

\author{
Alex Smith Araque Solano \\ Yuly Paola Silva
}

" DOI: https://doi.org/10.18601/01245996.v20n39.11. Este artículo es el resultado de la investigación Mercado inmobiliario y precios del suelo. Recepción: 13-01-2017, modificación final: 12-04-2018, aceptación: 11-05-2018. Sugerencia de citación: Araque S., A. S. y Silva, Y., P. (2018). La dinámica de la construcción en Bogotá 1995-2013. Revista de Economía Institucional, 20(39), 257-279.

a Profesor, Escuela de Economía, Universidad Sergio Arboleda, Bogotá, Colombia, [alex.araque@usa.edu.co].

b Economista, Escuela de Economía, Universidad Sergio Arboleda, Bogotá, Colombia, [yuly.silva@correo.usa.edu.co]. 


\section{La dinámica de la construcción en Bogotá 1995-2013}

Resumen. Los precios de la vivienda en Bogotá en el periodo 1995-2013 generaron un debate sobre la existencia de una burbuja inmobiliaria. Este artículo analiza los determinantes de la oferta y la demanda del sector, de los mercados asociados y de la velocidad de crecimiento de la tasa de interés y de los precios del suelo. Los resultados muestran que en el mercado no hubo una burbuja en 2012 y que las condiciones económicas de la década de 2010 provocaron el aumento de los precios de la vivienda. $\mathrm{E} 1$ aumento de estos precios en todo el país exige evaluar los efectos territoriales de la política nacional de vivienda, el papel de las administraciones locales, el uso de los instrumentos de gestión del suelo y los efectos de estas presiones de precios sobre la calidad y habitabilidad de las viviendas.

Palabras clave: burbuja inmobiliaria, precios del suelo; JEL: R11, R14, R31.

\section{The dynamics of building in Bogotá 1995-2013}

Abstract. The trends in housing prices in Bogotá in the period 1995-2013 generated a debate among experts about the existence of a real estate bubble. This paper analyzes the determinants of the supply and demand of the construction sector, the associated markets, and the rate of growth of interest rates and land prices. The results show that there was no bubble in the market in 2012 and that the economic conditions of the 2010 decade caused the increase in housing prices. The increase of these prices throughout the country requires the evaluation of the territorial effects of the national housing policy, the role of local administrations, the use of land management instruments and the effect of these price pressures on quality and habitability of housing.

Keywords: Real estate bubble, land prices; JEL: R11, R14, R31.

\section{A dinâmica da construção em Bogotá 1995-2013}

Resumo. Os preços de moradia em Bogotá no período 1995-2013 geraram um debate sobre a existência de uma bolha imobiliária. Este artigo analisa os determinantes da oferta e da demanda do setor, dos mercados associados e da velocidade de crescimento da taxa de juros e dos preços do solo. Os resultados mostram que, no mercado, não houve uma bolha em 2012 e que as condições econômicas da década de 2010 provocaram o aumento dos preços da moradia. $\mathrm{O}$ aumento desses preços, em todo o país, exige avaliar os efeitos territoriais da política nacional de habitação, o papel das administrações locais, o uso dos instrumentos de gestão do solo e os efeitos dessas pressões de preços sobre a qualidade e habitabilidade das moradias.

Palavras-chaves: bolha imobiliária, preços do solo; JEL: R11, R14, R31. 
- $\mathrm{n}$ las últimas décadas, los precios del sector de la construcción Cen Bogotá, en especial el segmento de vivienda, han alcanzado niveles históricos. Por ello, el análisis de los precios ha tomado fuerza en la discusión académica sobre los factores que han desencadenado la valorización en el mercado. Las preocupaciones por la presencia de una burbuja inmobiliaria local como la ocurrida en el país con el acelerado incremento de precios en los primeros años de la década de 1990 hanestado en el centro de la discusión desde los eventos acaecidos en la economía mundial en 2008 (Kalmanovitz, 2013; Cediel y Velásquez, 2015; Gómez et al., 2013).

En efecto, el comportamiento del precio real de las viviendas nuevas para el periodo 1995-2012 muestra un incremento de precios con una tendencia alcista en el largo plazo, lo que llama la atención por la capacidad de los hogares de la ciudad para soportar la subida de precios. El patrón alcista no es diferente para la vivienda usada ni para el canon de arrendamiento. En el primer caso, no solo se ha observado un rápido crecimiento de precios, sino también una reducción en la brecha de precios respecto a la vivienda nueva. En el segundo caso, los arrendamientos se ajustan con respecto al valor de las nuevas viviendas dirigidas a inversión, aunque su crecimiento es regulado.

Para el análisis académico es importante preguntar si la tendencia creciente de largo plazo en los productos inmobiliarios responde a la misma dinámica de sus determinantes, o si se explica por procesos especulativos de corto plazo. Por ello, este artículo busca determinar si el notable crecimiento de los precios de la vivienda desde 2003 respondió a sus fundamentales o si, en cambio, se trató de una burbuja inmobiliaria. En Bogotá, los determinantes son los factores constructivos incluido el suelo, el cual tiene particularidades importantes, dada la estructura urbana y la escasez relativa de tierra urbanizable. Las condiciones de demanda se observan por medio del PIB de la ciudad, un indicador robusto del ingreso de los hogares, y a partir de este, la disposición a pagar productos inmobiliarios según sus atributos: localización, proximidad a servicios urbanos, etc.

Mediante el uso de técnicas econométricas se busca establecer si la tendencia de los precios y su velocidad guardan relación con los cambios en sus fundamentales, siguiendo a Abad (2013), Froot y Maurice (1991), Agnello y Schuknecht (2009) y Harding y Pagan (2002).

El artículo se divide en cuatro secciones: la primera contiene los lineamientos teóricos. La segunda presenta los principales hechos de la dinámica reciente del sector de la vivienda a nivel nacional y en Bogotá. En la tercera sección se exponen los principales resultados 
econométricos sobre la posible existencia de una burbuja. Por último se presentan las conclusiones, discutiendo la tendencia del mercado en la ciudad.

\section{BURBUJA EN EL MERCADO INMOBILIARIO}

En la literatura no existe un concepto único de burbuja (Lind, 2008). Esta sección describe el concepto de burbuja como un modelo básico que explica cuándo la variación de los precios de un activo inmobiliario no corresponde al comportamiento de los fundamentales de la economía. A partir de la noción de activos y la posibilidad de transarlos en el mercado, una burbuja es el proceso en que los precios siguen un patrón de incremento sostenido para luego caer súbitamente. Desde la perspectiva de un inversionista, la burbuja se manifiesta cuando los precios son altos debido únicamente a la creencia de que serán más altos en el futuro (Stiglitz, 1990). Es decir, el precio del activo se incrementa en forma significativa sin el soporte de sus fundamentales, o determinantes, debido a fuerzas especulativas que mantienen expectativas alcistas de precios y rendimientos del activo. Otro síntoma de la presencia de una burbuja es la dinámica de los costos de construcción y de los servicios asociados al mercado inmobiliario (principalmente arrendamientos), que no siguen la tendencia alcista a la misma velocidad de crecimiento de los precios (Shiller, 2007).

En resumen, el patrón de precios de los productos inmobiliarios en presencia de una burbuja tiende a ser explicado más por las condiciones de capitalización del valor del activo que por las condiciones intrínsecas de este que pueden formar su valor real. Por tanto, la valorización financiera directa o indirecta de los productos inmobiliarios así como el desempeño de indicadores bursátiles pueden explicar más las variaciones de los precios e incluso dar con la especulación en el mercado inmobiliario (Shiller, 2007). En este sentido, cuestiones como la aversión al riesgo, la racionalidad del inversionista y la incertidumbre en los mercados pueden explicar el patrón de precios durante la burbuja.

Según Barkley, Rosser y Gallegati (2012), las burbujas pueden ser clasificadas en tres tipos dependiendo de su evolución y fin. En el primer tipo de burbuja, el activo sufre un aumento acelerado en sus precios, alcanza máximos, para luego caer de manera abrupta y "fuerte", volviendo a los fundamentales (materias primas); en el segundo tipo, los precios del activo alcanzan niveles máximos que se mantienen durante un tiempo, luego descienden y, en algunos casos en 
la misma proporción en la que subieron; en el tercer tipo de burbuja, el aumento exagerado de los precios es seguido por una reducción gradual de los mismos que persiste por un periodo corto, para luego caer de manera dramática a causa del pánico (mercados financieros).

Para explicar los cambios de precios de los productos inmobiliarios se expone un modelo básico de burbujas, en el que los fundamentales del mercado inmobiliario son los flujos de rentas del activo o su costo de uso. Siguiendo la variante del modelo lineal de valoración de activos (LAPM) propuesto por Glaeser y Nathanson (2015), se supone un precio de la vivienda único, que pagan todos los compradores y se determina sin arbitraje ${ }^{1}$. En estas condiciones, el valor actual de la vivienda debe ser igual a la renta neta actual que se obtiene de ella sumada a la valoración futura del activo, como en la ecuación 1:

$P_{t}=R_{t}+\frac{E\left(P_{t+1}\right)}{1+r}$

donde $P_{t}$ es el precio en $t, R_{t}$ el beneficio neto de la propiedad en el mismo periodo y $E\left(P_{t+1}\right)$ la expectativa del precio de la vivienda en el periodo siguiente, descontada a la tasa $r$. En este contexto, los fundamentales del precio se representan mediante la ecuación 2:

$E\left(\sum_{j=0}^{\infty} \frac{R_{t+j}}{(1+r)^{j}}\right)$

Los valores de $R_{t+j}$ se forman por la vía del mercado e incluyen la valoración de amenidades, la localización en la estructura urbana y el ingreso de los hogares. Si $R_{t}$ está gobernado por un proceso estocástico, el análisis de reversión a la media o exceso de volatilidad (estacionariedad) determinará la presencia o no de una burbuja inmobiliaria. En la forma básica de análisis ${ }^{2}$ se puede escribir el proceso para los beneficios netos como en la ecuación 3:

$R_{t}=\left(1+g_{r}\right) R_{t-1}+\in_{t}^{R}$

En este caso, $R_{t}$ exhibe una dinámica temporal de sí misma a una tasa de crecimiento constante $(g$,$) y un error i.i.d \in_{t}^{R}$. Cuando se resuelve el modelo para el precio de la vivienda se obtiene que:

$P_{t}=\frac{(1+r) R_{t}}{r-g_{r}}$

${ }^{1}$ Las condiciones de arbitraje se revisan en Stiglitz (2010).

${ }^{2}$ El proceso se presenta a manera de ilustración. Para una ampliación a marcos más sofisticados, ver Glaeser y Nathanson (2015). 
Lo cual implica que, ante pequeñas variaciones de los beneficios y tasas de interés favorables, los precios de la vivienda aumentarán de manera sustancial. Esta conclusión ilustra claramente lo que ocurre con los precios si los fundamentales cambian poco (o nada) mientras las condiciones anexas al mercado lo hacen vía tasas de interés, lo que lleva a que el precio refleje más estos cambios que los que están intrínsecamente relacionados con la generación y usufructo de un producto inmobiliario como la vivienda. Un resultado adicional radica en que la razón de desviaciones estándar del cambio en el precio y el cambio en la renta es la misma razón precio renta, por lo que el proceso es una caminata aleatoria con deriva.

Profundizando el análisis de la dinámica de los precios se puede revisar la forma en que varía la velocidad de estos con el fin de establecer condiciones para observar una burbuja tanto en niveles como en tasas de crecimiento. Siguiendo a Blanchard y Fischer (1993), en términos de tasas de beneficio, en equilibrio de mercado, se tiene que:

$\frac{E\left(p_{t+1} \vdots I_{t}\right)-p_{t}}{p_{t}}+\frac{d_{t}}{p_{t}}=r_{t}$

donde la expectativa de crecimiento del precio está condicionada a un conjunto de información $I_{t}$. Sumado al crecimiento de los precios se tiene un dividendo por la venta de la vivienda expresado como $d_{t}$. Bajo las condiciones de mercado en equilibrio, la suma de las tasas asociadas a la vivienda debe igualar a la tasa de interés $r_{t}$. Al multiplicar ambos lados de la ecuación por los precios en $t$ y dividir igualmente a ambos lados por la tasa de interés se tiene la siguiente expresión:

$a E\left(p_{t+1} \vdots I_{t}\right)+a d_{t}=p_{t}$

donde

$a=\frac{1}{1+r}<1$

La estabilidad de precios del activo exige que la tasa de descuento $|\mathrm{a}|$ $<$ 1. Si la ecuación (6) se cumple para todo $t$, se puede expresar así:

$p_{t}=\Sigma_{i=0}^{T} a^{i} E\left(d_{t+i} \vdots I_{t}\right)+a^{T+1} E\left(p_{t+T+1} \vdots I_{t}\right)$

Ahora bien, en el largo plazo, el límite del precio del activo será:

$p_{t}=\sum_{i=0}^{T} a^{i} E\left(d_{t+i} \vdots I_{t}\right)$

Como en el modelo LAPM, esto significa que el precio en cualquier momento del tiempo es la suma esperada de retornos o dividendos 
futuros, dado el conocimiento de la información existente en ese momento. La implicación de interés para este artículo es lo que ocurre en el corto plazo con la ecuación (5), pues diferenciándola con respecto a $t$ se puede analizar la velocidad de cada uno de sus componentes. Así se tiene que la velocidad de crecimiento de los precios de los inmuebles y de la renta del inmueble debería ser igual a la de la tasa de interés. Si los retornos crecen a mayor velocidad, habrá un desajuste sin respaldo, es decir, una burbuja inmobiliaria. En resumen, bien sea que el nivel de precios se aleje tendencialmente de sus fundamentales, o que su velocidad de crecimiento supere a la de la tasa de interés, en el corto plazo se tendrá un efecto de burbuja inmobiliaria.

Ahora bien, la vivienda es un bien con características particulares (altamente heterogéneo y difícilmente transable) diferentes de los activos financieros (Glaeser et al., 2008), y su oferta es relativamente inelástica. Por ello es de esperar una dinámica cíclica del mercado, que terminaría de configurar el patrón de la burbuja de precios (Steiner et al., 2012).

\section{DETERMINANTES DEL MERCADO INMOBILIARIO}

En esta sección se muestran los principales hechos que se observaron en el periodo de análisis, para el mercado inmobiliario nacional con base en la información del Censo de Obras del Dane. Esto con el fin de obtener una perspectiva de mediano plazo de la evolución del sector de la construcción, en particular del estado de la edificabilidad en las principales ciudades y áreas metropolitanas de Colombia.

Uno de los fenómenos que se observaron en otras economías ante la aparición de burbujas inmobiliarias fue la capacidad de la transmisión de fenómenos entre distintas ciudades (Shiller, 2007). Por esta razón no se puede estudiar de manera aislada a Bogotá sin tener en cuenta la dinámica nacional. La gráfica 1 muestra las cifras de Bogotá, Medellín, Cali y Bucaramanga, las cuatro ciudades donde se registró el 94\% del área en construcción en el primer trimestre de 2006 y del 90\% en apartamentos en el tercer trimestre de 2012.

E1 análisis de estas series revela: primero, que Bogotá representa cerca del 50\% del área en ejecución en el periodo, y en 2012 tiene el mismo nivel de participación que en 2006; segundo, que Medellín perdió participación y muestra un comportamiento simétrico con Bogotá, una situación similar a la de Cali y Bucaramanga; tercero, que la participación de Bogotá presenta una tendencia creciente en los dos primeros años y luego una suave tendencia decreciente, con 
trimestres donde hay una acentuada fluctuación desde 2011. Es decir, la dinámica inmobiliaria de Bogotá no ha sido diferente a la de las principales ciudades considerando su participación en el total construido.

Gráfica 1

Obras de apartamentos en ejecución

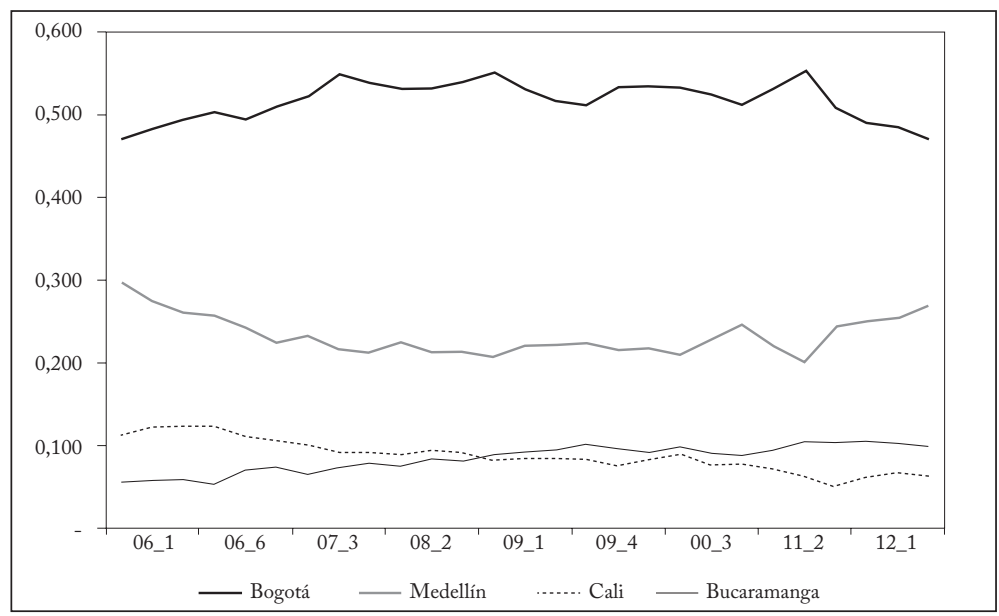

Fuente: Censo de obras del Dane.

Por otro lado, si bien el área en apartamentos creció al 2,7\% trimestral, pierde participación en el área total construida, es decir, en este periodo hubo un auge de oficinas y de otros productos como bodegas, instalaciones de educación, hoteles, hospitales y administración pública. La tasa mencionada de crecimiento de los apartamentos, en Bogotá y en el agregado nacional, sugiere, entre otras cosas, que las preferencias de productos inmobiliarios han cambiado, a una mayor demanda de apartamentos, y que la escasez y el alto valor del suelo urbanizable en las ciudades analizadas, que se resumen en el precio del suelo para construcción, reducen las áreas construibles, como predicen los modelos estándar de economía urbana ${ }^{3}$.

Lo anterior se puede observar en la proporción entre áreas de construcción de casas y apartamentos, que funciona como una proxy del precio del suelo edificado ${ }^{4}$. Asumiendo que la reducción del área construida en casas obedece al alto costo de oportunidad frente a la

3 Para una descripción detallada de estos modelos, ver Glaeser (2008).

${ }^{4}$ En 2006-2012, la correlación negativa anual entre esta proporción y el Índice de Valoración Predial del Dane es: Medellín 0,92; Cali 0,95; Barranquilla 0,52; Bucaramanga: 0,94 y total nacional: 0,52. 
edificación en altura, la gráfica 2 muestra las proporciones en Bogotá, Barranquilla, Medellín, Bucaramanga, Cali y en el total nacional.

Gráfica 2

Proporción del área de casas y apartamentos

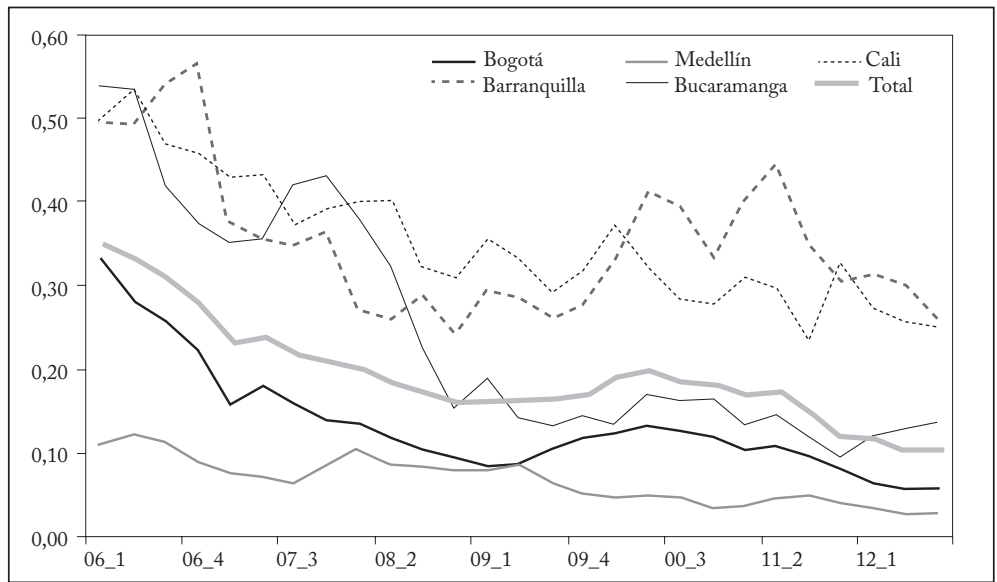

Fuente: Censo de Obras del Dane, elaboración propia.

La gráfica 2 muestra que en todas las ciudades hay una tendencia decreciente en distintos grados, lo que sugiere que el precio del suelo ha aumentado. Los casos de Medellín y Bogotá son notorios, por cuanto en la primera la proporción era de 0,10 y en Bogotá de poco más de 0,30 en 2006, mientras que en el tercer trimestre de 2012 se redujo a 0,03 y 0,06 respectivamente, inferior a la del total nacional. La situación en Bucaramanga es muy parecida, pues la proporción se redujo unos 40 puntos en solo siete años.

Se puede inferir que el precio del suelo urbano en Colombia tuvo un incremento notable en el pasado reciente, no solo en Bogotá; de hecho, en las ciudades intermedias se observa una reducción del área en casas, lo que sugiere un aumento notable del costo marginal de producción de vivienda y, por supuesto, de su precio. Si bien la dinámica en conjunto es similar, como es de esperar, los precios no lo son en niveles. Aunque los precios aumentaron, ninguna ciudad se equipara a Bogotá. Y no hay evidencia de un efecto de contagio de subidas de precios en la dinámica observada en Bogotá (Enríquez, Campo y Avendaño, 2015). 


\section{MERCADOS DE LA CONSTRUCCIÓNY DEL SUELO EN BOGOTÁ}

La situación de altos precios de la vivienda en Bogotá se puede entender como un desequilibrio del mercado, donde la oferta no logra satisfacer la demanda, como sugiere el mayor ritmo de crecimiento de la demanda con respecto al de la oferta. Cuando esto sucede se genera escasez y esta presiona los precios al alza, como muestra el estudio de Steiner et al. (2012)5. En lo que resta del artículo, el análisis se centra en los factores de oferta que determinan los altos precios de la vivienda observados en Bogotá.

\section{Gráfica 3}

Logaritmo del área de construcción licenciada, 1995-2012

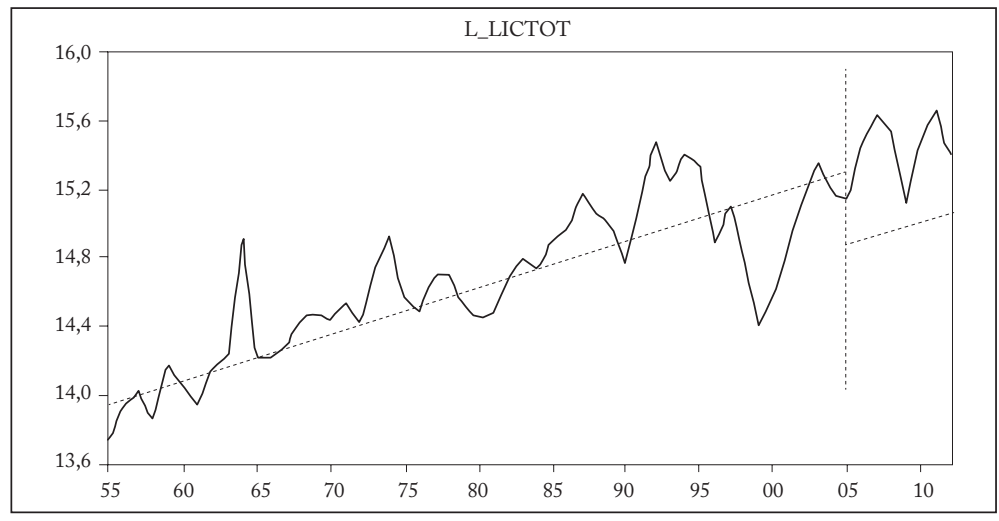

Fuente: ELIC: Dane; elaboración propia.

La revisión del área licenciada, un indicador del ritmo de la oferta de vivienda, muestra un menor crecimiento. Este hecho observado en la producción se puede explicar a partir de los principales determinantes de la oferta: precio del suelo, costos de construcción, salarios y costos de financiación; junto al entorno institucional.

En la gráfica 4, que muestra la evolución de los precios, se separaron los tres últimos quintiles para hacer visible la atipicidad de los precios del quintil de hogares de mayor ingreso. Se pueden observar, además, tres periodos: aumento de precios en la segunda mitad de

${ }^{5}$ Cediel y Velásquez (2015) señalan que la oferta de vivienda en estratos bajos no compensa el nivel de formación de hogares, por lo que aumenta el déficit cuantitativo. Mientras que la mayor cantidad de vivienda nueva vendida fue para estratos 5 y 6 , sus precios aumentaron más rápido que la economía del país, lo cual coincide con nuestros resultados (la velocidad de crecimiento de los precios de la vivienda para quintiles 4 y 5 es mayor que la de la tasa de interés). 
la década de 1990, reducción en 1997-2002 y crecimiento sostenido en 2003-2012.

Gráfica 4

Precio real del metro cuadrado
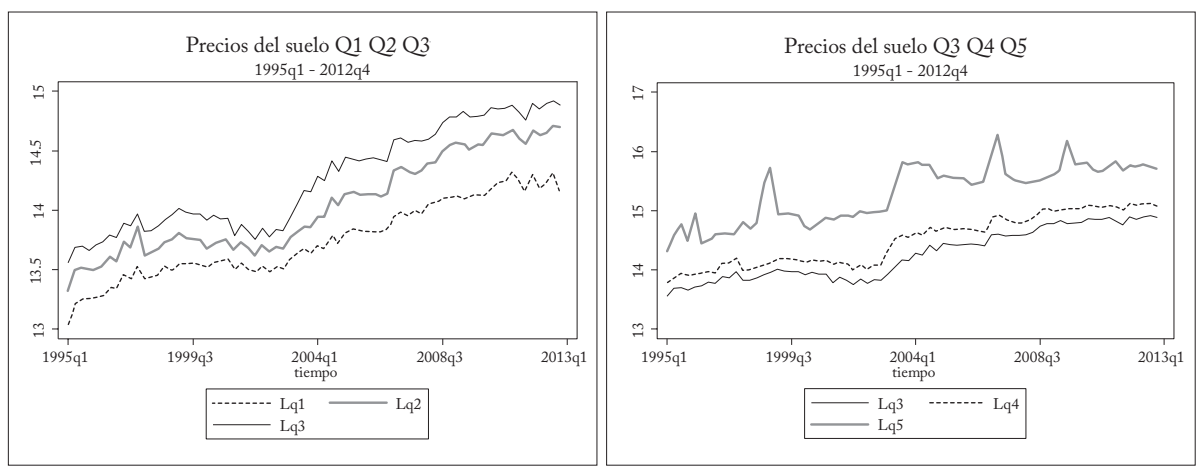

Fuente: Revista La Guía, elaboración propia.

En la gráfica se observa que la crisis tocó fondo alrededor de 2003, cuando se inició un proceso de rápido aumento del precio, salvo en el último quintil, donde parece crecer en forma sostenida todo el periodo.

Cuadro 1

Tasa anual de crecimiento por quintil

\begin{tabular}{lccccc}
\hline Periodo & Quintil 1 & Quintil 2 & Quintil 3 & Quintil 4 & Quintil 5 \\
\hline $1995-2002$ & 0,047 & 0,030 & 0,023 & 0,029 & 0,058 \\
$2003-2012$ & 0,074 & 0,100 & 0,093 & 0,077 & 0,058 \\
\hline
\end{tabular}

Fuente: Revista La Guía, cálculos propios.

El cuadro 1 muestra que el crecimiento anual de los precios del metro cuadrado fue del 4,7\%, el 3,0\%, el 2,3\%, el 2,9\% y el 5,8\% anual en los quintiles 1, 2, 3, 4 y 5, respectivamente, en el periodo 1995-2002. En el periodo 2003-2012, el crecimiento se aceleró en todos los quintiles, excepto en el último donde se mantuvo en el 5,8\% anual, lo que induca un aumento estable del valor del metro cuadrado. En los demás grupos de hogares hubo crecimientos superiores al $7 \%$ para los quintiles 1 y 4 , y superiores al 9\% para los quintiles 3 y 2 , del 10\% para este último.

\section{ESTIMACIÓN DE LA BURBUJA INMOBILIARIA EN BOGOTÁ}

Para detectar la presencia o no de una burbuja inmobiliaria se hace un análisis econométrico para probar dos hipótesis. En primer lugar, 
se busca establecer si hay una tendencia común entre los precios de la vivienda, el precio del suelo y el PIB de Bogotá ${ }^{6}$, la cual probaría que el precio se forma en función de sus determinantes.

En segundo lugar, se pretende probar que la velocidad de crecimiento de los precios no ha superado la de las tasas de interés de referencia, lo que desmontaría cualquier indicio de burbuja porque los choques de corto plazo se desvanecerían y la serie retornaría a su tendencia.

\section{ANÁLISIS EXPLORATORIO}

En el cuadro 2 se presentan los resultados de las regresiones que intentan captar los cambios de ritmo del crecimiento en 1995-2012, para los cinco quintiles.

Cuadro 2

Ritmo de crecimiento del precio del metro cuadrado, 1995-2012

\begin{tabular}{lccccc}
\hline Variable & Quintil 1 & Quintil 2 & Quintil 3 & Quintil 4 & Quintil 5 \\
\hline \multirow{2}{*}{ bp_02 } & 0,0066 & 0,0167 & 0,0167 & 0,0115 & $-0,0063$ \\
& 0,0002 & $1,76 \mathrm{e}-11$ & $7,28 \mathrm{e}-10$ & $2,72 \mathrm{e}-06$ & 0,2766 \\
\hline \multirow{2}{*}{ d_02 } & $-1,1367$ & $-2,7890$ & $-2,6708$ & $-1,6753$ & 1,5353 \\
& $1,42 \mathrm{e}-04$ & $1,71 \mathrm{e}-11$ & $2,70 \mathrm{e}-09$ & $3,23 \mathrm{e}-05$ & 0,1148 \\
\hline \multirow{2}{*}{ tiempo } & 0,0116 & 0,0073 & 0,0058 & 0,0071 & 0,0142 \\
& $9,56 \mathrm{e}-12$ & 0,0001 & 0,0044 & 0,0004 & 0,0043 \\
\hline \multirow{2}{*}{ cons } & 11,6504 & 12,5249 & 12,9512 & 12,9582 & 12,6097 \\
\hline \multirow{2}{*}{ R2 } & $3,80 \mathrm{e}-57$ & $2,51 \mathrm{e}-53$ & $7,50 \mathrm{e}-51$ & $6,57 \mathrm{e}-52$ & $5,06 \mathrm{e}-26$ \\
\hline
\end{tabular}

Fuente: Revista La Guía, cálculos propios.

La primera columna presenta las variables; la primera variable es el break point en 2002 (bp_02), resultado del producto de una variable dicótoma de ceros antes de 2002 y unos hasta el último trimestre de 2012, la segunda variable, una dummy de 2002 (d_02), y la tercera es la variable de tiempo.

Como se ve en todas las columnas, el crecimiento trimestral fue sostenido en todos los quintiles de precios, pero en el primero y el último la tasa de crecimiento fue notablemente superior a la de los otros tres quintiles, es decir, mientras que el precio del metro cuadrado de vivienda en el primero y en el último quintil creció al 1,16\% y al $1,42 \%$ en el primer periodo, en los otros tres quintiles creció como

${ }^{6}$ En Colombia, Lauchlin Currie (1993) inició la tradición de utilizar el sector de la construcción como sector líder del crecimiento (Montenegro, 2012; DNP, 1972); después se lo utilizó en periodos de crisis como mecanismo de recuperación. Loría et al. (2012) corroboran la existencia de asociación entre el sector de la construcción y la tasa de desempleo. 
máximo al 0,73\%, cerca de un punto porcentual por trimestre. En el periodo final, el crecimiento es inverso, al menos para el primer quintil.

Las estimaciones indican que en algunas zonas el crecimiento del precio del suelo fue superior al $6 \%$ anual en todo el periodo. Los cuadros 3, 4 y 5 muestran los resultados de las regresiones. En la primera columna aparece el nombre de la variable y en las siguientes las estimaciones de la tasa de crecimiento anual (year) y de la constante (_cons), acompañada del valor $p$ en cada zona.

Cuadro 3

Zonas con crecimiento de precio de suelo superior al $6 \%$

Comercio e industria

\begin{tabular}{lcccccc}
\hline \multirow{2}{*}{ Variable } & Ciudad Salitre & Tramo 4 \\
& & Carrera 15 & E1 Lago \\
Calles 114 a & Country & Usaquén & Fontibón & Toberín \\
& 127 & & & & \\
\hline \multirow{2}{*}{ year } & 0,0713 & 0,0667 & 0,0644 & 0,0693 & 0,0621 & 0,0786 \\
& $1,37 \mathrm{e}-60$ & $1,75 \mathrm{e}-22$ & 0,00026 & 0,00001 & $9,62 \mathrm{e}-09$ & $7,88 \mathrm{e}-15$ \\
\hline \multirow{2}{*}{ cons } & $-128,4011$ & $-118,8791$ & $-114,8152$ & $-124,313$ & $-110,4766$ & $-144,0606$ \\
& $6,35 \mathrm{e}-06$ & $1,81 \mathrm{e}-20$ & 0,00083 & 0,00005 & $7,35 \mathrm{e}-08$ & $9,16 \mathrm{e}-14$ \\
\hline $\mathrm{R}^{2}$ & 0,6445 & 0,8477 & 0,4473 & 0,5858 & 0,7675 & 0,8168 \\
\hline
\end{tabular}

Fuente: Lonja de Propiedad Raíz de Bogotá (LPRB), cálculos propios.

E1 cuadro 3 agrupa las zonas según la clasificación por uso económico de la LPRB: la zona comercial del Centro Distrital de Negocios (CDN) y de Ciudad Salitre, de comercio para hogares de altos ingresos como el tramo 4 de la carrera 15 entre calles 114 y 127, la zona de E1 Lago-Country y de Usaquén. También figura una zona de comercio popular, Fontibón, y la zona industrial de Toberín. Si bien la presión sobre los precios del suelo no es homogénea, no discrimina por sectores económicos; aunque donde hay una intensa actividad económica la renta del suelo es alta y también su precio.

E1 cuadro 4 agrupa las zonas residenciales: Cedritos, de familias de ingresos medios; Sur Oriente 3, una zona residencial popular; La Cabrera, Rosales, Chicó Reservado, Multicentro-Santa Bárbara Central, Santa Bibiana-San Patricio-Navarra, Santa Bárbara Occidental, de familias de altos ingresos; y Ciudad Salitre y La Colina Campestre, zonas residenciales de estrato medio alto. La de mayor crecimiento es Ciudad Salitre, al 7,4\% anual; seguida por Sur Oriente 3 , al 7,2\% anual.

De acuerdo con Lauchlin Currie (1993) existe una relación directa entre urbanización y alto ingreso per cápita, así como entre expansión del mercado y tasa de urbanización. Estas dos relaciones concuerdan con las mayores tasas de crecimiento de los precios del 
suelo en las zonas donde el uso comercial e industrial es intensivo o de alta jerarquía, y en zonas residenciales de hogares de altos ingresos. Según Currie, el desarrollo de las ciudades es "económico", es decir que las personas que se quieran beneficiar estarán cerca de la actividad económica, sin importar el precio que deban pagar por el suelo; de modo que el nivel de vida y la urbanización dependen del ingreso per cápita (ibíd., p. 236).

Cuadro 4

Zonas con crecimiento de precio de suelo superior al 6\%

Residencial

\begin{tabular}{|c|c|c|c|c|c|}
\hline Variable & Cedritos & Sur Oriente 3 & La Cabrera & Rosales & $\begin{array}{c}\text { Chicó } \\
\text { Reservado }\end{array}$ \\
\hline \multirow{2}{*}{ year } & 0,0711 & 0,0719 & 0,0671 & 0,0639 & 0,0675 \\
\hline & $1,76 \mathrm{e}-23$ & 0,0568 & $3,71 \mathrm{e}-29$ & $1,88 \mathrm{e}-29$ & $2,31 \mathrm{e}-06$ \\
\hline \multirow[b]{2}{*}{ _cons } & $-128,681$ & $-132,136$ & $-119,440$ & $-113,244$ & $-120,691$ \\
\hline & $8,58 \mathrm{e}-22$ & 0,0701 & $5,93 e-27$ & $3,64 \mathrm{e}-27$ & $1,14 \mathrm{e}-05$ \\
\hline $\mathrm{R}^{2}$ & 0,877 & 0,752 & 0,916 & 0,919 & 0,628 \\
\hline Variable & $\begin{array}{c}\text { Multicentro } \\
\text { Sta Bárbara } \\
\text { Central } \\
\end{array}$ & $\begin{array}{c}\text { Santa Bibiana } \\
\text { San Patricio } \\
\text { Navarra }\end{array}$ & $\begin{array}{c}\text { Santa Bárbara } \\
\text { Occidental }\end{array}$ & Ciudad Salitre & $\begin{array}{c}\text { Colina } \\
\text { Campestre }\end{array}$ \\
\hline \multirow{2}{*}{ year } & 0,0625 & 0,0654 & 0,0644 & 0,0745 & 0,0694 \\
\hline & $9,46 \mathrm{e}-27$ & $7,96 \mathrm{e}-31$ & $4,16 e-30$ & $3,42 \mathrm{e}-07$ & $2,99 \mathrm{e}-08$ \\
\hline \multirow{2}{*}{ _cons } & $-110,912$ & $-116,824$ & $-114,811$ & $-135,166$ & $-125,285$ \\
\hline & $1,53 \mathrm{e}-24$ & $1,18 \mathrm{e}-28$ & $6,40 \mathrm{e}-28$ & $1,55 \mathrm{e}-06$ & $1,70 \mathrm{e}-07$ \\
\hline $\mathrm{R}^{2}$ & 0,906 & 0,928 & 0,923 & 0,684 & 0,744 \\
\hline
\end{tabular}

Fuente: LPRB, cálculos propios.

Gráfica 5

Licencias de construcción y PIB nacional

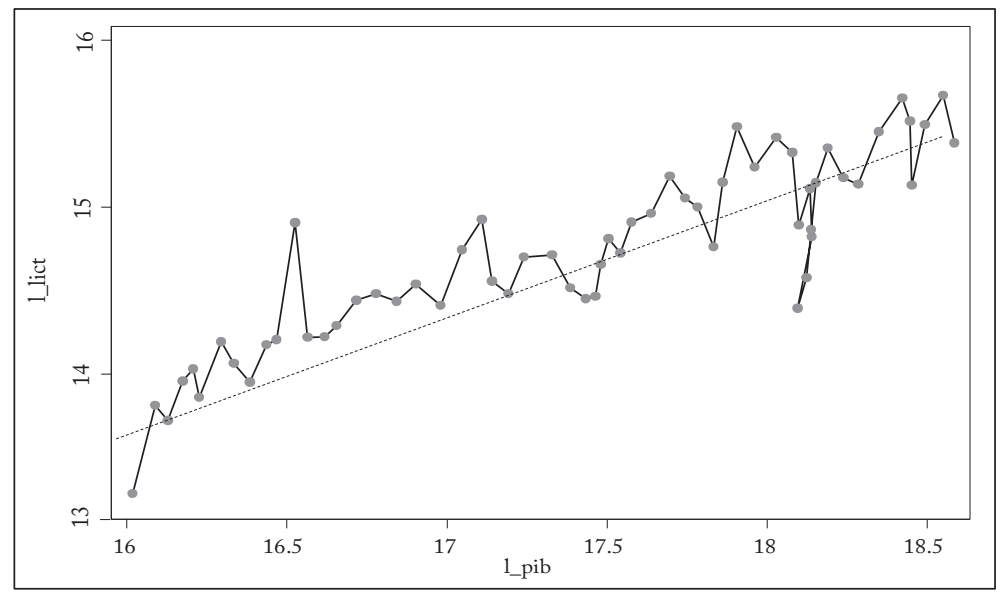

Fuente: ELIC: Dane. PIB: Greco, elaboración propia. 
Como ya se mencionó, es necesario revisar la relación entre construcción y actividad económica para determinar si la demanda es respaldada por el "crecimiento económico". Como se observa en la gráfica 5, cuyo eje horizontal es el PIB y el vertical las licencias de construcción, la relación de largo plazo es estable. La pendiente de la línea de tendencia indica que el aumento de la actividad económica va acompañado de un aumento de menor proporción en la construcción. La tasa de crecimiento del PIB en el periodo es del 4,5\% mientras que la del área licenciada es del 3,5\%.

La gráfica 6, que representa las tasas de crecimiento del PIB y de las licencias de construcción, muestra que en algunos años la actividad del sector creció más rápido que la actividad económica y su relación tiende a alejarse del equilibrio de largo plazo, pero años después, en general cuatro, retorna al equilibrio. Una situación similar se presenta en las crisis aunque el intervalo es menor, de modo que la tasa de crecimiento del PIB se mueve en forma casi paralela a la del sector.

Gráfica 6

Licencias de construcción y tasa de crecimiento del PIB

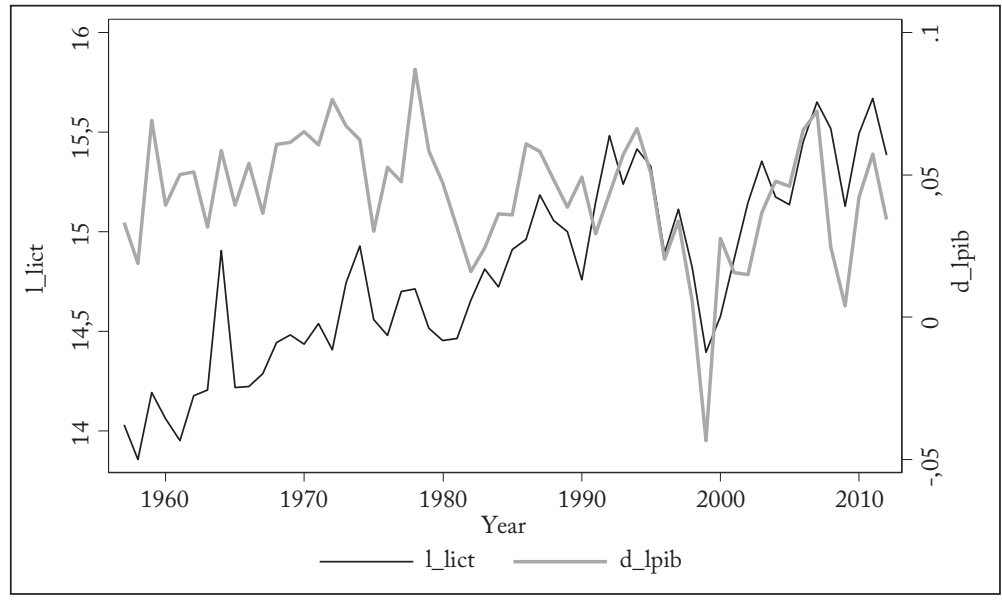

Fuente: ELIC: Dane. PIB: Greco, elaboración propia.

Además, se pueden distinguir dos periodos: 1955-1984 y 1985-2012. En el primero, los movimientos de las series son menos sincrónicos que en el segundo, donde el sector se mueve de acuerdo con las fluctuaciones del PIB. Se podría decir que la evolución del sector, si no está determinada por el desempeño económico, al menos su dinámica es muy similar, es decir, la oferta es determinada principalmente por la actividad económica. El cuadro 6 presenta los resultados de las 
pruebas de causalidad de Granger entre licencias de construcción y PIB en una secuencia de cinco rezagos.

La primera columna muestra las hipótesis: la actividad constructora no causa el PIB en el sentido de Granger y el PIB no causa la actividad constructora en el sentido de Granger, en las demás se presenta el valor $p$ asociado a cada hipótesis en el rezago establecido. En el primer rezago no hay evidencia para rechazar la hipótesis nula, de modo que la actividad constructora causa el PIB con un rezago, pero de ahí en adelante no se puede rechazar la hipótesis de no causalidad. Por su parte, el PIB causa la actividad constructora en el sentido de Granger en los dos primeros rezagos, y del tercero al quinto no hay evidencia de causalidad. En el tercero la evidencia no es contundente para rechazar la hipótesis nula, aunque si se aumenta el nivel de significancia el PIB causa la actividad constructora.

Cuadro 5

Causalidad de Granger: PIB y actividad constructora

\begin{tabular}{lccccc}
\hline Hipótesis & R1 & R2 & R3 & R4 & R5 \\
\hline L_LICTOT no causa L_PIB & 0,0191 & 0,5656 & 0,6081 & 0,6075 & 0,7961 \\
L_PIB no causa L_LICTOT & 0,0009 & 0,0044 & 0,0842 & 0,1967 & 0,3409 \\
\hline
\end{tabular}

Fuente: Dane, cálculos del autor.

¿Cómo entender estos resultados? Si se parte de un periodo de crisis, las medidas para reactivar la construcción tendrán un efecto multiplicador en toda la actividad económica, cuyo efecto se diluye en el segundo periodo posterior a la reactivación. El impacto en la economía tiene un efecto de búmeran en la construcción, por retroacción, es decir, en el segundo periodo la economía jalona y sostiene la actividad edificadora.

Este tipo de causalidad se debe reflejar en la evolución de largo plazo de las dos series y la poder constatar una prueba cointegración, como en efecto la constatan las pruebas de Johansen. La información estadística sugiere la existencia de al menos un vector cointegrante, de modo que en una regresión de las dos variables existe una combinación lineal cuyos residuos son estacionarios ${ }^{7}$. En otras palabras, en el largo plazo la construcción y el PIB muestran una relación estable que puede ser afectada por algunos eventos económicos, que alejan esta relación del equilibrio de largo plazo, aunque con el tiempo retorna al equilibrio. Para probar esta hipótesis se estimó un modelo donde las licencias dependen del PIB, de la brecha del PIB con respecto a su

7 El estadístico de prueba es 27,31 y el valor crítico al 5\% es 15,41. 
potencial y del patrón temporal de los residuos, el cual se representa en la ecuación (9).

llictot $t_{t}=\beta_{0}+\beta_{1} l P I B_{t}+\beta_{2}$ gappib $+\sum_{i=0}^{p} \gamma_{i} \mu_{t-1}+\sum_{j=1}^{q} \alpha_{j} e_{t-j}$

E1 cuadro 6 presenta los resultados. E1 coeficiente del logaritmo del PIB indica que un incremento del $100 \%$ en la actividad económica induce un aumento del $57 \%$ en el área de construcción licenciada. La desviación del PIB con respecto a su tendencia de largo plazo tiene un efecto importante. Si el PIB aumenta un punto porcentual más que el PIB potencial, las licencias aumentan un 3,7\%. Así, si el área licenciada normal en Bogotá fuera de 5 millones de metros cuadrados al año y la tasa de crecimiento del PIB nacional fuera mayor en un punto porcentual, el área licenciada aumentaría en 185 mil metros cuadrados debido a este aumento de la actividad económica por encima del PIB potencial.

Cuadro 6

Licencias de construcción

\begin{tabular}{lcc}
\hline Variable & Coeficiente & Valor $p$ \\
\hline Constante & 4,7608 & 0,030 \\
L_PIB & 0,5733 & 0,000 \\
GAP_LPIB & 3,7126 & 0,022 \\
AR(1) & 0,7721 & 0,000 \\
MA(2) & $-0,2777$ & 0,039 \\
\hline R $^{2} 0,9168$ & \\
\hline Raíces AR invertidas & 0,77 \\
Raíces MA invertidas & 0,53 & $-0,53$ \\
\hline
\end{tabular}

Fuente: ELIC: Dane; PIB: Greco; cálculos propios.

En suma, aumentos mayores del PIB llevan a que el sector de la construcción tienda a crecer más de lo normal. Ahora bien, el componente autorregresivo muestra la inercia del sector ante su propia dinámica, es decir, la actividad edificadora se desacelera o acelera lentamente porque lo que ocurre en el periodo inmediatamente anterior determina lo que ocurrirá en el periodo actual. Por su parte, el componente de media móvil indica que el sector solo internaliza parte de los choques en el segundo periodo después de que ocurran.

\section{LA CONSTRUCCIÓN, EL PIB Y LOS PRECIOS DEL SUELO}

Estudios anteriores analizan el comportamiento de los precios de la vivienda para determinar si realmente existe una burbuja en el mercado inmobiliario. Entre las pruebas más destacadas se encuentra la de raíz unitaria propuesta por Phillips, Wu y Yu (2007) y Phillips, Shi y Yu 
(2012), que Gómez et al. (2013) aplicaron al mercado colombiano. Por su parte, Cediel y Velásquez (2015) utilizan un modelo VEC estructural para estudiar la relación de largo plazo entre precios de la vivienda, precios del suelo y PIB.

La mayoría de los estudios que analizan el comportamiento de los precios de la vivienda no tienen en cuenta el precio ni la escasez del suelo como determinantes (Ciro, 2015), los cuales afectan a la ciudad porque aumentan la densificación y el precio del metro cuadrado de tierra (Marulanda, 2013). Se estimaron varias regresiones para determinar la tasa de crecimiento de largo plazo de los precios del suelo en todas las zonas de la LPRB, establecer la dinámica de precios en toda la ciudad e identificar las zonas indicativas del precio general del suelo.

La actividad constructora está determinada por la evolución de la actividad económica, los insumos y los precios del suelo. Los insumos -materiales y mano de obra- también evolucionan a la par de las condiciones económicas, mientras que el precio del suelo es una variable residual: del precio de venta del espacio edificado se descuenta el pago de los factores y lo que queda se destina a pagar el suelo, y presenta diferencias en cada zona debidas al ingreso y las preferencias de localización de los hogares, como ya vimos. La relación entre la construcción, el PIB y el precio del suelo se estimó mediante una regresión de las licencias de construcción, el PIB nacional y los precios en distintas zonas.

Debido a la distinta la longitud de las series, a que hay referentes sobre la evolución del precio del suelo y su efecto sobre la actividad constructora, se consideraron las siguientes zonas residenciales y comerciales: Centro Internacional Bavaria, Chapinero, Avenida Chile, San Victorino, E1 Ejido-Gorgonzola, La Cabrera, Los Rosales, Alhambra, Niza Norte, Cedritos, Nueva Santa Fe, Suroriente 2, y Bosa Residencial. Los resultados de las estimaciones con corrección de Prais Winsten se presentan en el cuadro 7 , que muestra los coeficientes y el valor $p$ de las variables en cada zona.

El mayor crecimiento de los precios del suelo se observa en Cedritos, seguido por La Cabrera y Los Rosales. Los precios del suelo no son afectados por la actividad constructora; los afecta el PIB, como parece lógico, aunque su presión no es homogénea. Asimismo, los precios del suelo no determinan la evolución de la construcción; en cambio, la actividad económica tiene efectos sobre la evolución del sector.

Como ya se señaló, puesto que el precio del suelo es una variable residual y no debería afectar el desempeño normal de la actividad constructora; si el precio del terreno hiciera parte de los precios 
Cuadro 7

Precio del suelo, actividad constructora y PIB

\begin{tabular}{|c|c|c|c|c|c|c|c|c|}
\hline Variable & $\begin{array}{c}\text { Centro Int. } \\
\text { Bavaria }\end{array}$ & Chapinero & \multicolumn{2}{|c|}{ Av. Chile } & \multicolumn{2}{|c|}{ San } & $\begin{array}{c}\text { E1 Ejido } \\
\text { Gorgonzola }\end{array}$ & $\begin{array}{c}\text { La } \\
\text { Cabrera }\end{array}$ \\
\hline \multirow{2}{*}{ L_lict } & 0,055 & 0,006 & \multicolumn{2}{|c|}{0,020} & \multicolumn{2}{|c|}{0,021} & 0,0499 & $-0,028$ \\
\hline & 0,574 & 0,941 & \multicolumn{2}{|c|}{0,833} & \multicolumn{2}{|c|}{0,839} & 0,507 & 0,754 \\
\hline & 0,232 & 0,359 & \multicolumn{2}{|c|}{1,369} & \multicolumn{2}{|c|}{0,625} & 0,681 & 1,586 \\
\hline & 0,499 & 0,213 & \multicolumn{2}{|c|}{$3,50 \mathrm{E}-10$} & \multicolumn{2}{|c|}{0,097} & 0,004 & $4,25 \mathrm{E}-11$ \\
\hline \multirow{2}{*}{ _cons } & 9,653 & 8,136 & \multicolumn{2}{|c|}{$-10,144$} & \multirow{2}{*}{\multicolumn{2}{|c|}{$\begin{array}{l}3,236 \\
0,610\end{array}$}} & 0,043 & $-13,515$ \\
\hline & 0,103 & 0,100 & \multicolumn{2}{|c|}{0,001} & & & 0,991 & $8,90 \mathrm{E}-05$ \\
\hline $\mathrm{R}^{2}$ & 0,899 & 0,921 & \multicolumn{2}{|c|}{0,940} & \multicolumn{2}{|c|}{0,875} & 0,940 & 0,941 \\
\hline Variable & $\begin{array}{c}\text { Los } \\
\text { Rosales }\end{array}$ & Alhambra & $\begin{array}{l}\text { Niza } \\
\text { Norte }\end{array}$ & \multicolumn{2}{|c|}{ Cedritos } & $\begin{array}{c}\text { Nueva } \\
\text { Santa Fe }\end{array}$ & $\begin{array}{l}\text { Surorien- } \\
\text { te } 2\end{array}$ & $\begin{array}{l}\text { Bosa } \\
\text { Resid. }\end{array}$ \\
\hline \multirow{2}{*}{ L_lict } & $-0,052$ & 0,013 & \multirow{2}{*}{$\begin{array}{l}0,002 \\
0,982 \\
\end{array}$} & \multicolumn{2}{|c|}{$-0,005$} & $-0,077$ & 0,040 & 0,020 \\
\hline & 0,602 & 0,844 & & \multicolumn{2}{|c|}{0,952} & 0,216 & 0,357 & 0,810 \\
\hline \multirow{2}{*}{ L_PIB } & 1,570 & 1,394 & \multirow{2}{*}{$\begin{array}{c}1,158 \\
3,57 \mathrm{E}-07\end{array}$} & \multirow{2}{*}{\multicolumn{2}{|c|}{$\begin{array}{c}2,137 \\
1,62 \mathrm{E}-11\end{array}$}} & $-0,073$ & 0,897 & 1,314 \\
\hline & $5,76 \mathrm{E}-13$ & $3,95 \mathrm{E}-12$ & & & & $7,26 \mathrm{E}-01$ & $5,91 \mathrm{E}-11$ & $3,92 \mathrm{E}-09$ \\
\hline \multirow[b]{2}{*}{ _cons } & $-13,146$ & $-11,888$ & \multirow{2}{*}{$\begin{array}{c}-7,587 \\
0,021\end{array}$} & -25 & & 14,748 & $-4,666$ & $-11,851$ \\
\hline & $1,07 \mathrm{E}-05$ & $2,77 \mathrm{E}-05$ & & 2,28 & -07 & $1,29 \mathrm{E}-04$ & 0,011 & $2,04 \mathrm{E}-04$ \\
\hline $\mathrm{R}^{2}$ & 0,940 & 0,958 & 0,981 & & & 0,960 & 0,989 & 0,978 \\
\hline
\end{tabular}

Fuente: LPRB; PIB: Greco; cálculos propios.

de producción del espacio construido, su aumento determinaría la dinámica de la actividad, pero no es así, como señala la teoría de la renta del suelo. El precio del terreno es aquel que se está dispuesto a pagar en una zona localizada a cierta distancia del centro o del CDN. La relación entre localización y precio se establece para un nivel de utilidad dado (Alonso, 1964).

La serie de precios de la vivienda se construyó con los datos de la Revista La Guía desde 1995 hasta el primer trimestre de 2013. Por la dificultad para localizar los proyectos en cada zona y usar precios por estratos, debido a que la Unidad Administrativa Especial de Catastro Distrital hizo al menos un cambio de nomenclatura en el periodo, se construyeron quintiles de precios y se examinó su dinámica de largo plazo. La gráfica 7 muestra los cambios de precios y de la tasa de interés.

El ejercicio permite distinguir dos periodos: en el primero, de entre 1992 a 1997, hubo un notable aumento de los precios de la vivienda que generó una burbuja inmobiliaria, la cual estalló en 1998 y llevó a la crisis del sector hasta comienzos de 2002 (ANIF, 2011). En el segundo, de 2010 a 2013 se observa rápido aumento de los precios de la vivienda.

En el caso de las viviendas de precio bajo, Q1, desde mediados de 1995 hasta finales de 1997 la tasa de crecimiento de los precios fue superior a la de la tasa de interés. En 2008-2013 la tasa de interés aumentó más que la de los precios de la vivienda. Así sucedió en los demás quintiles, excepto en el quinto. El cuadro 8 muestra el creci- 
miento acumulado de la tasa de interés y de los precios de la vivienda y la conclusión es contundente. Entre 1995 y 1997 el crecimiento acumulado de la tasa de interés fue negativo, es decir, la tasa de interés se redujo mientras que los precios de la vivienda aumentaron. En 2010-2012 el acumulado de la tasa de interés fue mayor que el de todos los precios, excepto el de viviendas de precio alto. Es decir, hubo un notorio crecimiento de precios, soportado por factores económicos.

\section{Gráfica 7}

Velocidad de los precios de la vivienda y del crecimiento del DTF (por quintiles)
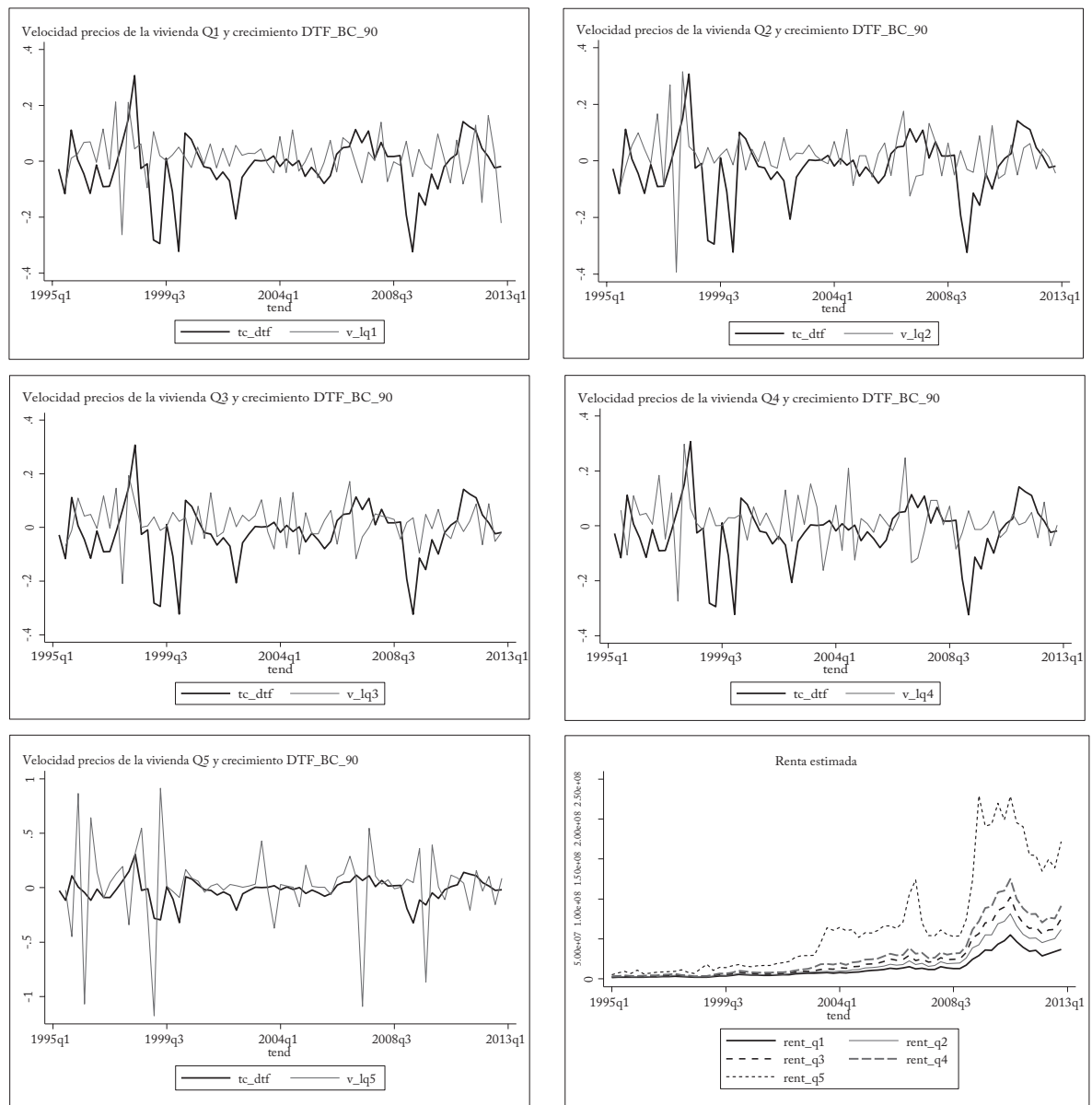

Fuente: Revista La Guía, Banco de la República, cálculos propios. 
Cuadro 8

Acumulado de la velocidad de crecimiento

\begin{tabular}{lllllll}
\hline & Tasa de interés & Q1 & Q2 & Q3 & Q4 & Q5 \\
\hline $1995-1997$ & $-0,188$ & 0,208 & 0,116 & 0,235 & 0,076 & 0,408 \\
\hline $2010-2012$ & 0,267 & $-0,054$ & 0,046 & 0,077 & 0,079 & 0,491 \\
\hline
\end{tabular}

Fuente: Revista La Guía, Banco de la República, cálculos propios.

\section{CONCLUSIONES}

La evolución de los precios de la vivienda en el periodo 1995-2013 muestra la dinámica y la turbulencia del sector, así como la corrección de los desajustes en el largo plazo. La burbuja de precios de la primera parte de la década de 1990 es un referente para evaluar la existencia de otra burbuja en la década de 2010. El análisis de los fundamentales del sector, de la dinámica de los precios y de la tasa de interés señala que en este último periodo no hubo una burbuja inmobiliaria.

La evidencia muestra que las fuerzas de la economía tienden a llevar al sector al equilibrio de largo plazo. Las desviaciones de la tasa de crecimiento del PIB afectan el comportamiento normal del sector de la construcción. Los precios del suelo y los costos de construcción no determinan la evolución de la actividad constructora, lo que tiene efectos sobre la evolución del sector es la actividad económica. Como parece lógico, el PIB afecta el precio del suelo. Por ello, las expectativas sobre el futuro de la economía son centrales en la demanda de vivienda.

Es importante señalar que los precios de la vivienda usada se incrementan con los de la vivienda nueva; el canon de arrendamiento y el índice de precios de vivienda usada muestran el mismo comportamiento de largo plazo. En todas las ciudades hubo un crecimiento de los precios del suelo, no solo en Bogotá. Y puesto que fue inducido por la actividad económica, que se traduce en ingresos, es necesario evaluar el papel de las administraciones locales y sus instrumentos de intervención. Las administraciones locales no cumplen un papel pasivo en la provisión y la calidad de la vivienda.

\section{REFERENCIAS BIBLIOGRÁFICAS}

Abad, J. (2013). Precios de la vivienda: sobrevaloración y burbuja, [www. ucm.es/data/cont/docs/518-2013-10-23-Abad13.pdf].

Agnello, L. y Schuknecht, L. (2009). Booms and busts in housing markets. Determinants and implications. ECB working paper 1071, [https:// papers.ssrn.com/sol3/papers.cfm?abstract_id=1433198]. 
Alonso, W. (1964). Location and land use. Toward a general theory of land rent. Londres: Harvard University Press.

ANIF. (2011). El crédito hipotecario en Colombia: evaluación del impacto regulatorio pos-crisis, [http://anif.co/sites/default/files/investigaciones/ anif-bid0511.pdf].

Banco de la República de Colombia. (2013). Informe de estabilidad financiera. Bogotá: Banco de la República.

Banco de la República. (s.f.). Tasas de interés. Colombia, [www.banrep. gov.co/es/tasas-interes].

Barkley, J., Rosser, M. y Gallegati, M. (2012). A Minsky-Kindleberger perspective on the financial crisis. Journal of Economic Issues, 46(2), 449-458.

Blanchard, O. y Fischer, S. (1993). Lectures on macroeconomics. Cambridge, Mass.: The MIT Press.

Cediel, V. y Velásquez, C. (2015). ¿Hay una burbuja inmobiliaria en Bogotá? Un estudio por segmentos de mercado. Revista de Economía Institucional, 17(32), 233-257.

Ciro, J. A. (2015). ¿Hay o no burbuja inmobiliaria en Bogotá? ¿Cómo va el consenso?, [www.eafit.edu.co/escuelas/economiayfinanzas/noticiaseventos/Paginas/burbuja-inmobiliaria-en-Bogota.aspx].

Currie, L. (1993). La política urbana en un marco macroeconómico. Cuadernos de economía, 13(18-19), 233-248.

Currie, L. (1993). La teoría en que se basa la estrategia del sector líder. Cuadernos de economía, 13(18-19), 225-230.

Dane. (2011). Estadísticas por tema: Construcción, [www.dane.gov.co/ index.php/estadisticas-por-tema/construccion/censo-de-edificaciones].

DNP. (1972). Las cuatro estrategias. Bogotá: DNP, [https://colaboracion. dnp.gov.co/CDT/PND/Pastrana1_Prologo.pdf].

Enríquez H., Campo, J. y Avendaño, A. (2015). Relaciones regionales en los precios de vivienda nueva en Colombia. Ecos de Economía, 19(40), 25-47.

Froot, K. y Maurice, O. (1991). Intrinsic bubbles: The case of stock prices. American Economic Review, 81(5), 1189-1214.

Glaeser, E., Gyourko, J. y Saiz, A. (2008). Housing supply and housing bubbles. NBER working paper 14193. Cambridge, Mass.

Glaeser E. (2008). Cities, agglomeration and spatial equilibrium. Oxford: Oxford University Press.

Glaeser E. y Nathanson, C. (2015). Housing bubbles. En G. Duranton, V. Henderson y W. Strange (eds.). Handbook of regional and urban economics (pp. 701-752) Oxford, UK: Elsevier.

Gómez, J. et al. (2013). Testing for bubbles in housing markets: New results using a new method, [https://www.dallasfed.org/ /media/ documents/institute/wpapers/2013/0164.pdf].

Harding, D. y Pagan, A. (2002). Dissecting the cycle: A methodological investigation. Journal of Monetary Economics, 49(2), 365-381, [www. sciencedirect.com/science/article/pii/S0304393201001088].

Jaramillo, S. y Cuervo, N. ( 2014). Precios inmobiliarios de vivienda en Bogotá 1970-2013. Documentos CEDE. Bogotá: Uniandes. 
Kalmanovitz, S. (2013). ¿Burbuja inmobiliaria?, [www.elespectador.com/ opinion/burbuja-inmobiliaria-columna-435097].

Lind, H. (2008). Price bubbles in housing markets. International Journal of Housing Markets and Analysis, 2(1), 78-90.

Loría, E., Libreros, C. y Salasti, E. (2012). Crisis de paro en España: una aplicación de la ley de Okun, Coyuntura Económica, 42(2), 135-152.

Marulanda, G. (2013). Falta de suelo presiona costo de vivienda: Catastro, [www.eltiempo.com/archivo/documento/CMS-12721975].

Montenegro, Á. (2012). Lauchlin Currie: desarrollo y crecimiento económico. Revista de Economía Institucional, 14(27), 81-97.

Phillips, P., Wu, Y. y Yu, J. (2007). Explosive behavior in the 1990's Nasdaq: When did exuberance escalate asset values? HKIMR working paper 22/2007, [www.hkimr.org/uploads/publication/175/ ub_full_0_2_164_hkimr-no22_bw.pdf].

Phillips, P., Yu, J, y Shi, S.-P. (2012). Testing for multiple bubbles. Cowles. discussion paper 1843, New Haven, Conn.

Shiller, R. (2007). Understanding recent trends in house prices and home ownership. NBER working paper 13553, Cambridge, Mass.

Steiner, R., Salazar, N. et al. (2012). ¿Qué tan desalineados están los precios de la vivienda en Colombia? Bogotá: Fedesarrollo.

Stiglitz, J. E. (1990). Symposium on bubbles. Journal of economic perspectives, 4(2), 13-18. 